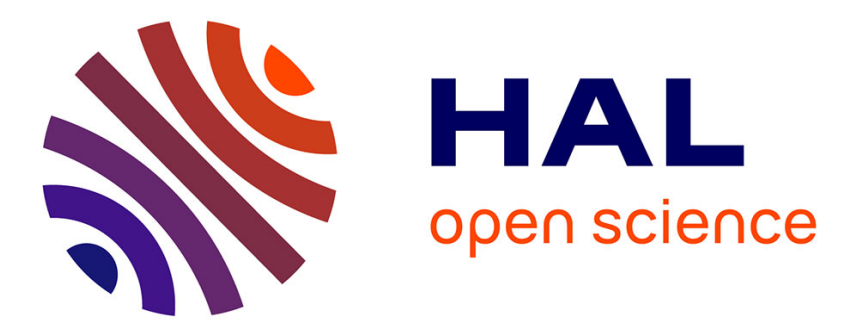

\title{
Tuning Mie Scattering Resonances in Soft Materials with Magnetic Fields
}

Thomas Brunet, Kevin Zimny, Benoit Mascaro, Olivier Sandre, Olivier

Poncelet, Christophe Aristégui, Olivier Mondain-Monval

\section{- To cite this version:}

Thomas Brunet, Kevin Zimny, Benoit Mascaro, Olivier Sandre, Olivier Poncelet, et al.. Tuning Mie Scattering Resonances in Soft Materials with Magnetic Fields. Physical Review Letters, 2013, 111 (264301), pp.1-5. 10.1103/PhysRevLett.111.264301 . hal-00932072

\section{HAL Id: hal-00932072 \\ https://hal.science/hal-00932072}

Submitted on 26 Nov 2018

HAL is a multi-disciplinary open access archive for the deposit and dissemination of scientific research documents, whether they are published or not. The documents may come from teaching and research institutions in France or abroad, or from public or private research centers.
L'archive ouverte pluridisciplinaire HAL, est destinée au dépôt et à la diffusion de documents scientifiques de niveau recherche, publiés ou non, émanant des établissements d'enseignement et de recherche français ou étrangers, des laboratoires publics ou privés. 


\title{
Tuning Mie scattering resonances in soft materials with magnetic fields
}

\author{
Thomas Brunet, ${ }^{1, *}$ Kevin Zimny, ${ }^{2}$ Benoit Mascaro, ${ }^{1}$ Olivier Sandre, ${ }^{2}$ \\ Olivier Poncelet, ${ }^{1}$ Christophe Aristégui, ${ }^{1}$ and Olivier Mondain-Monval ${ }^{3}$ \\ ${ }^{1}$ Université de Bordeaux, Institut de Mécanique et d'Ingénierie, \\ CNRS UMR 5295, 351 boulevard de la Libération, 33405 Talence, France \\ ${ }^{2}$ Université de Bordeaux, Laboratoire de Chimie des Polyméres Organiques, \\ CNRS UMR 5629, 16 avenue Pey Berland, 33607 Pessac, France \\ ${ }^{3}$ Université de Bordeaux, Centre de Recherche Paul Pascal, \\ CNRS UPR 8641, 115 avenue du Docteur Schweitzer, 33600 Pessac, France
}

(Dated: October 12, 2013)

\begin{abstract}
An original approach is here proposed to reversibly tune Mie scattering resonances occurring in random media by means of external low induction magnetic fields. This approach is valid for all scalar fields of waves (either optical or acoustical). The experimental demonstration is supported by ultrasound experiments performed on emulsions made of fluorinated ferrofluid spherical droplets dispersed in a Bingham fluid. We show that the electromagnet-induced change of droplet shape into prolate spheroids, with a moderate aspect ratio of 2.5 , drastically affects the effective scattering properties of the disordered medium. Its effective acoustic attenuation coefficient is shown to vary by a factor of 5 , by controlling both the flux density and orientation of the applied magnetic field.

PACS numbers: 43.35.+d,43.20.+g,47.65.Cb, 82.70.Kj
\end{abstract}

Since the pioneer works of Gustav Mie [1], wave scattering has aroused a growing interest in physics [2], in particular with the intention of controlling wave propagation by taking benefit from scattering resonances as sought for metamaterials [3]. As predicted by theories on resonant multiple scattering of light [4] or sound waves [5] in the Mie scattering regime, i.e., when the wavelength within the particles is comparable to their diameter, the effective properties of random media are deeply altered nearby the particle frequency resonances. Since the size and the shape of the particles fix them, these resonant modes cannot be easily tuned with rigid particles $[6,7]$. By contrast, the use of all-fluid structures should be more appropriate to tune the scattering resonances, as fluid objects may easily be deformed under various external stimuli. Since a long time, it is indeed well known that immiscible fluid drops suspended in a fluid matrix can elongate under extensional hydrodynamic flows [8], electric fields [9] or magnetic fields [10]. Such changes in the particle shape should drastically affect the effective properties of random media as predicted for light [11] and for sound waves [12], paving the way for the realization of tunable and adaptive multifunctional materials.

For experimental studies on resonant wave scattering by an assembly of deformable particles, ultrasonic techniques present the advantages to readily detect the wave field (not just the intensity as in optics) and to resolve the measurements in both time and space. Moreover, the characteristic length scales (between $1 \mu \mathrm{m}$ and $1 \mathrm{~mm}$ ) of particles make the synthesis of systems of disordered resonant scatterers rather convenient over a broad range of scattering contrasts: bubbly media [13] or suspensions of solid spheres [14]. Other contrasted materials are emulsions made of fluorinated-oil droplets randomly dispersed in a water-based gel [15]. They were shown to exhibit a large collection of multipolar Mie resonances due to the sound-speed contrast between the particles and the host matrix. Such a model system of disordered resonant particles is easily achievable by means of microfluidics, which allows an accurate control of particle parameters: size, size dispersity, shape, and volume fraction [16, 17].

In this letter, we tuned the effective acoustic properties of resonant emulsions using external low induction magnetic fields. The latter are sufficient to elongate initially spherical ferrofluid droplets into prolate spheroids whose axis of rotational symmetry is along the magnetic field direction [18]. To do so, we doped fluorinated-oil droplets with superparamagnetic $\gamma-\mathrm{Fe}_{2} \mathrm{O}_{3}$ nanoparticles according to an original process leading to a stable fluorinated ferrofluid. Briefly, the proper dispersion of iron oxide nanoparticles in fluorinated oil involves the adsorption of a perfluoropoly(ether) surfactant onto the nanoparticles followed by a chemical grafting of a perfluorinated silane on iron oxide. The grafting efficiency and the colloidal stability of the nanoparticles were investigated by several methods including FT-IR and X-ray photoemission spectroscopy, and light and neutron scattering techniques [19]. As illustrated in Fig. 1, a single droplet of such fluorinated ferrofluid embedded in a water-based gel can elongate by a factor 2.5 under a magnetic field induction of $43 \mathrm{mT}$. During the application of a cycle of increasing and decreasing magnetic field inductions, the axial aspect ratio $a / b$ describes a hysteretic loop. This is due to the relaxation time of the interface between the droplet and the host gel matrix. However, the deformation is fully reversible as the droplet recovers its initial spherical shape after a sufficient time $(\sim 30 \mathrm{~min})$ after cancellation of the magnetic field. 


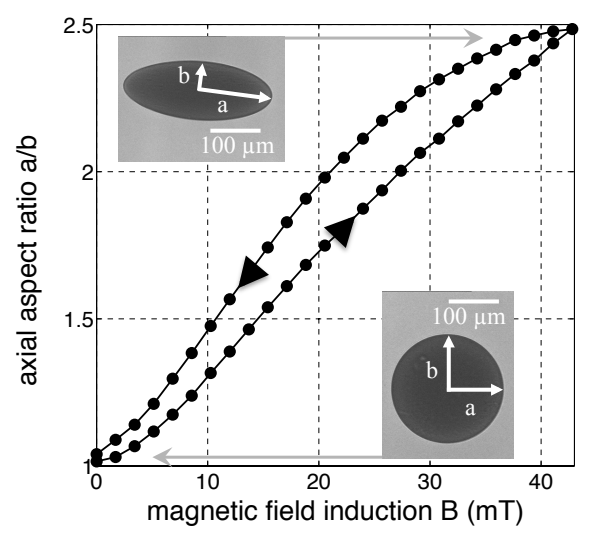

FIG. 1. Axial aspect ratio $a / b$ of a single fluorinated ferrofluid droplet embedded in a water-based gel matrix, submitted to a cycle of increasing and decreasing magnetic field inductions, with a 20-s delay between each step (a movie is available in the supplementary materials).

Materials and methods. - Robotics is the most appropriate method to produce highly monodisperse emulsions of such magnetic droplets [15]. In our experiments, the fluorinated ferrofluid is continuously injected through a fused silica capillary with internal/outer diameters of $75 / 150 \mu \mathrm{m}$, directly into a water-based gel (Carbopol@), which behaves like an acoustically transparent host matrix. In addition, the low yield stress $(\sim 1.5 \mathrm{~Pa})$ of this Bingham fluid circumvents the issue of sedimentation of the fluorinated ferrofluid droplets $\left(\rho_{1}=1.9\right.$ g.cm $\left.{ }^{-3}\right)$, which are denser than the host matrix $\left(\rho_{0}=1 \mathrm{~g} . \mathrm{cm}^{-3}\right)$. The injecting capillary moves within the gel matrix at a constant velocity of about $10 \mathrm{~mm} . \mathrm{s}^{-1}$ giving birth to perfectly identical spherical droplets of about $100 \mu \mathrm{m}$ in radius as shown in Fig. 2(a). The droplet-size distribution of the emulsion is optically characterized before applying the magnetic field providing a mean radius $\langle a\rangle$ of 105 $\mu \mathrm{m}$ and a coefficient of variation of $4 \%$ (Fig. 2(b)).
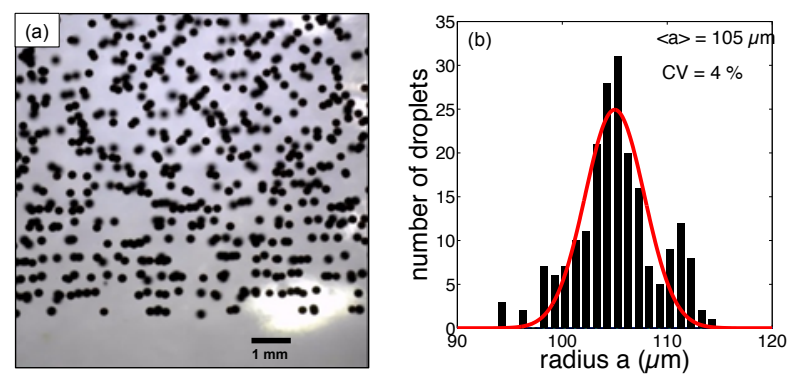

FIG. 2. (Color online) (a) Optical microscopy picture of fluorinated ferrofluid droplets embedded in a water-based gel matrix, achieved by means of robotics $\left(\Phi_{V} \sim 1 \%\right)$. (b) Corresponding histogram of the droplet-size distribution. Mean radius $\langle a\rangle=105 \mu \mathrm{m}$ and coefficient of variation $\mathrm{CV}=4 \%$.

The sample is then confined into a 5 mm-thickness acoustic measurement cell with small lateral dimensions $\left(30 \times 30 \mathrm{~mm}^{2}\right)$, on which a piezoelectric transducer of 5 $\mathrm{MHz}$ central frequency and of 0.50 inch diameter has been stuck as illustrated in Figs. 3. Since its surface is sufficiently large [20], the piezoelectric transducer measures the wave field averaged over its face. Moreover, the incoherent part of the acoustic field is negligible as we only consider dilute emulsions with volume fractions $\Phi_{V}$ of about $1 \%$, in order to avoid coalescence between droplets when they elongate under the external magnetic field. Thus, the remaining detected signal is the coherent wave $[7,14]$. The cell-transducer ensemble just takes up the width of the air gap $(40 \mathrm{~mm})$ of an electromagnet that generates a homogenous magnetic field of which induction can be precisely tuned up to $B=43 \mathrm{mT}$. The measurement cell can also be turned along with its vertical axis to vary the angle $\zeta$ between the direction of the magnetic field $B$ and the acoustic wave vector $k_{0}$ (Fig. $3(\mathrm{a}))$. Acoustic experiments are then performed using multi-echo ultrasound spectroscopy techniques allowing the accurate measurement of the effective acoustic attenuation coefficient of the emulsion [15].

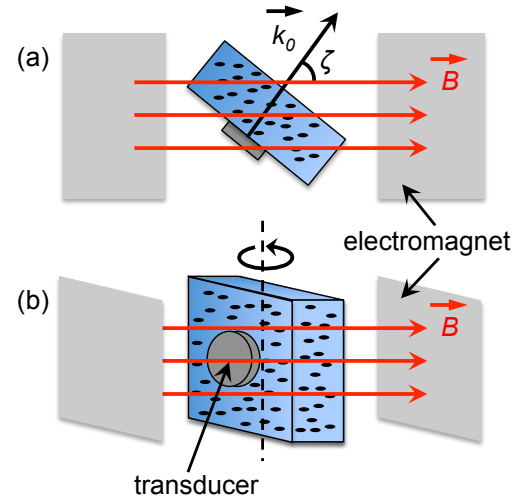

FIG. 3. (Color online) (a) Top and (b) side view of the experimental setup dedicated to acoustic measurements on fluorinated ferrofluid droplet emulsions under magnetic fields with various inductions (up to $43 \mathrm{mT}$ ) and orientations. The angle $\zeta$ between the direction of the magnetic field $B$ and the acoustic wave vector $k_{0}$ can be varied between $0^{\circ}$ and $90^{\circ}$.

Results.- A first acoustic experiment is performed on the fluorinated ferrofluid droplet emulsion without applying any magnetic field (thickest blue lines in Figs. 4). The attenuation spectra clearly exhibit several peaks that are due to the multipolar Mie resonances of the spherical droplets [15]. From such an acoustic resonant response, an inverse procedure [21] provides an acoustically measured mean radius $\langle a\rangle$ of $106 \mathrm{~m}$ and a coefficient of variation of $5 \%$, in excellent agreement with optical microscopy measurements (Fig. 2(b)). Then, the attenuation spectra are recorded for a cycle of increasing and decreasing magnetic field inductions with a 20-second delay between two acoustic measurements. When the direction of the applied magnetic field $B$ is parallel to 
the acoustic wave vector $k_{0}$ (Fig. $4(\mathrm{a})$ ), the attenuation spectrum tends to shrink down towards lower frequencies (Fig. 4(b)). By contrast, the attenuation peaks clearly shift towards higher frequencies (Fig. 4(e)) when the magnetic field $B$ is applied perpendicularly to the acoustic wave vector $k_{0}$ (Fig. $4(\mathrm{~d})$ ). In both configurations, the initial attenuation spectra are fully recovered after cancellation of the magnetic fields (Figs. 4(c) and (f)). As suggested by the hysteretic loop shown in Fig. 1, the initially spherical droplets become spherical again after being elongated into prolate spheroids with an axial aspect ratio of 2.5 for the highest magnetic field induction. Therefore, the effective acoustic parameters of the emulsion can be reversibly tuned over a broad range of values.
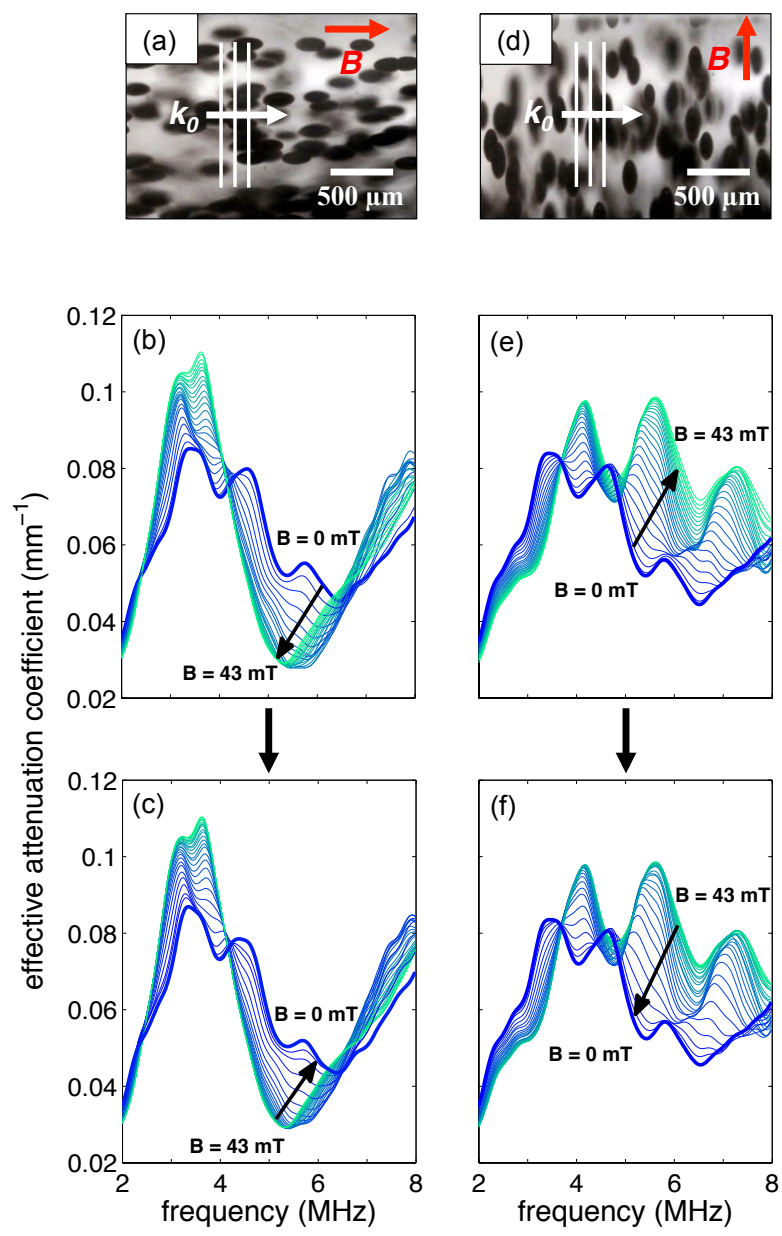

FIG. 4. (Color online) Evolution of the attenuation frequency spectra of fluorinated ferrofluid droplet emulsions submitted to a cycle of increasing ( $b$ and e) and decreasing (c and f) magnetic field inductions (from $0 \mathrm{mT}$ to $43 \mathrm{mT}$ ). The direction of the magnetic field $B$ is parallel ( $\mathrm{a}, \mathrm{b}$ and $\mathrm{c}$ ) or perpendicular $\left(\mathrm{d}\right.$, e and $\mathrm{f}$ ) to the incident acoustic wave vector $k_{0}$. Thickest curves refer to the reference emulsion made of spherical droplets (no magnetic field). Pictures (a and d) show the orientation of the fluorinated ferrofluid droplets along the direction of the magnetic field $B$ (movies are available in the supplementary materials).
Model. - Since the volume fraction of droplets for the considered emulsion is quite low $\left(\Phi_{V} \sim 1 \%\right)$, the experimental results are analyzed within the framework of the Independent Scattering Approximation. Indeed, crosscorrelations between scatterers have not to be taken into account contrary to more concentrated media [22] or random media made of strongly resonant particles such as air bubbles [13]. Thus, the effective attenuation coefficient $\alpha_{\text {eff }}$ of the coherent pressure wave propagating in our dilute emulsion is given by [23]:

$$
\alpha_{e f f}=\frac{2 \pi}{k_{0}} \int_{a} \eta(a) \operatorname{Im}\left[F_{a}(0)\right] \mathrm{d} a
$$

where $k_{0}$ is the incident wave-number in the host matrix, $\eta(a)$ is the number of $a$-radius droplets per unit volume, $\Phi=\int_{a} \eta(a) \times\left(4 \pi a^{3} / 3\right) \mathrm{d} a$ the total volume fraction of droplets, and $F_{a}(0)$ is the forward scattering function of a single droplet that depends on both the material properties of the particles and those of the host matrix. For a given couple of materials, the frequency locations of the resonant attenuation peaks are inversely proportional to the droplet size [15]. As the coefficient $\alpha_{e f f}$ depends on the forward scattering function $F_{a}(0)$, one can get an intuitive understanding of the evolution of the attenuation frequency spectra as function of the magnetic field induction (Figs. 4) by only considering the droplet size along with the incident acoustic wave vector $k_{0}$. When the magnetic field direction is parallel to the incoming acoustic waves, the droplets elongate along that direction (Fig. 4(a)) and the resonant attenuation peaks shift towards lower frequencies (Figs. 4(b) and (c)). Inversely, when the magnetic field is applied perpendicularly to the incoming acoustic waves, the droplet size become smaller along that direction (Fig. 4(d)) and the attenuation peaks shift towards higher frequencies (Figs. 4(e) and (f)).

To go beyond such a qualitative analysis, the forward scattering function $F_{a}(0)$ of the prolate droplets has to be derived in order to retrieve the effective attenuation coefficient measured in our emulsions. Wave scattering by spheroidal objects has been the subject of many theoretical works [24] and the exact solution of the acoustic wave scattering by a liquid prolate spheroid was obtained many decades ago [25]. From the latter theoretical work, the forward scattering function $F_{a}(0)$ of a prolate fluorinated ferrofluid droplet, whose axial aspect ratio $a / b$ is 2 , is calculated providing the effective attenuation coefficient of our emulsions submitted to an external magnetic induction $B_{0}$ of $25 \mathrm{mT}$ (see Fig. 1) for various orientations $\zeta$. Theoretical predictions and experimental measurements are compared for five angles $\zeta$ between the direction of the magnetic field $B$ and the acoustic wave vector $k_{0}: \zeta=$ $0^{\circ}\left(B_{0}\right.$ is parallel to $\left.k_{0}\right), 22.5^{\circ}, 45^{\circ}, 67.5^{\circ}$, and $90^{\circ}\left(B_{0}\right.$ is perpendicular to $k_{0}$ ) revealing an excellent quantitative agreement as shown in Fig. 5. 


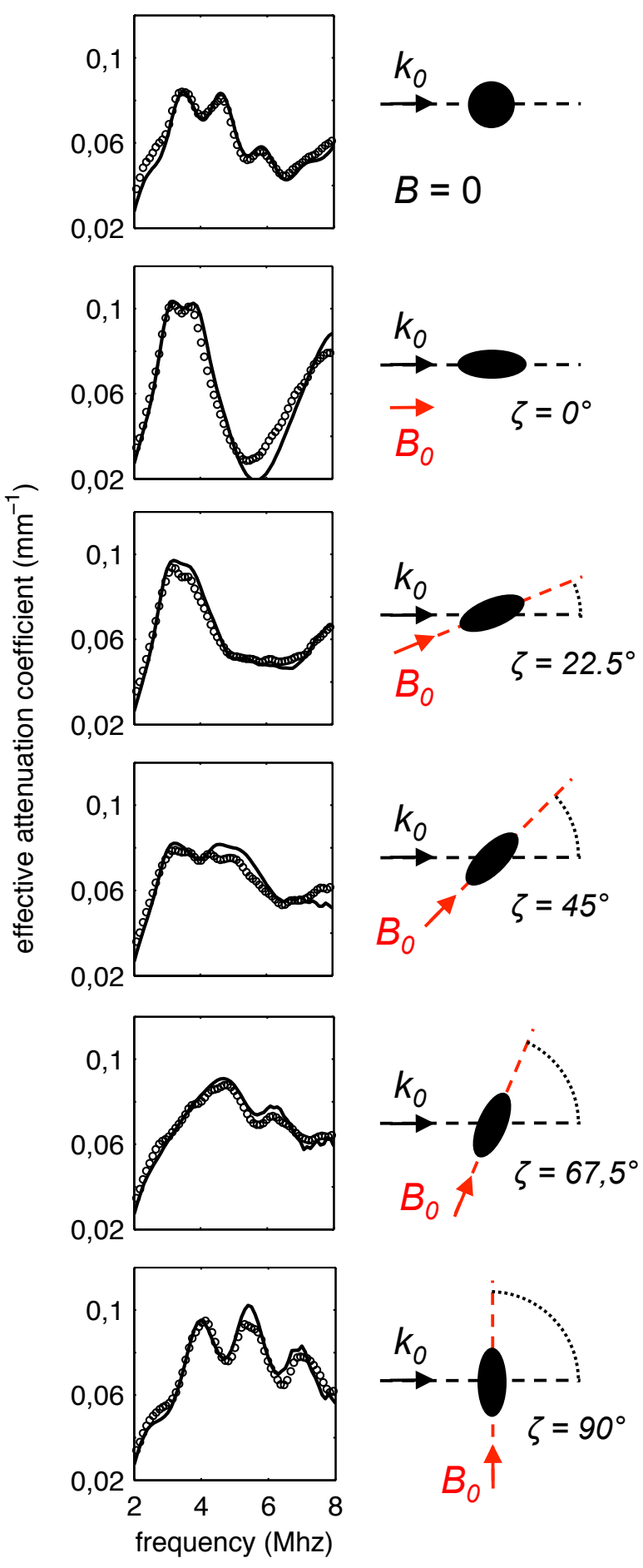

FIG. 5. (Color online) Comparison between the measured attenuation coefficients (circles) and those predicted in the framework of the Independent Scattering Approximation (solid lines) for emulsions made of prolate fluorinated ferrofluid droplets with an axial aspect ratio of 2 . Various orientations between the direction of the magnetic field (of $B_{0}$ $=25 \mathrm{mT}$ induction) and the acoustic wave vector $k_{0}$ are investigated $\left(\zeta=0^{\circ}, 22.5^{\circ}, 45^{\circ}, 67.5^{\circ}\right.$, and $\left.90^{\circ}\right)$. The top figure refers to the reference emulsion made of spherical droplets (no magnetic field).
In conclusion, the resonant attenuation in our emulsion can be widely tuned (by a factor 5) by controlling both the flux density and orientation of external magnetic fields. Such a tunability of the resonant properties of scattering media should be of the utmost relevance for metamaterials, of which unusual properties are driven by scattering resonances [3]. As reported for electromagnetic waves, the use of magnetic fields turned out to be efficient to tune the effective permeability of metamaterials made of split ring resonators [26]. This external stimulus should also be appropriate to tune the properties of locally resonant materials in acoustics [27], providing large enough mechanical contrasts between the resonant particles and the host matrix as expected with soft acoustic metamaterials [28].

* thomas.brunet@u-bordeaux1.fr

[1] G. Mie, Ann. Phys.-Berlin, 25, 377 (1908).

[2] P. Sheng, Introduction to Wave Scattering, Localization, and Mesoscopic Phenomena (Springer, Heidelberg, 2006).

[3] C. M. Krowne and Y. Zong, eds., Physics of Negative Refraction and Negative Index Materials (Springer, Berlin, 2007).

[4] A. Lagendijk and B. A. van Tiggelen, Phys. Rep., 270, 143 (1996)

[5] R. E. Challis, M. J. W. Povey, M. L. Mather, and A. K. Holmes, Rep. Prog. Phys., 68, 1541 (2005).

[6] H. P. Schriemer, M. L. Cowan, J. H. Page, P. Sheng, Z. Liu, and D. A. Weitz, Phys. Rev. Lett., 79, 3166 (1997)

[7] A. Derode, A. Tourin, and M. Fink, Phys. Rev. E, 64, 036605 (2001).

[8] G. I. Taylor, Proc. R. Soc. Lond. A, 138, 41 (1932)

[9] C. T. O'Konski and H. C. Thacher, J. Phys. Chem., 57, 955 (1953).

[10] V. I. Arkhipenko, Y. D. Barkov, and V. G. Bashtovoi, Magnetohydrodynamics, 14, 373 (1979).

[11] S. Asano, Appl. Opt., 18, 712 (1979).

[12] A. S. Ahuja and W. R. Hendee, J. Acoust. Soc. Am., 63, 1074 (1978).

[13] V. Leroy, A. Strybulevych, J. H. Page, and M. G. Scanlon, Phys. Rev. E, 83, 046605 (2011).

[14] M. L. Cowan, J. H. Page, and P. Sheng, Phys. Rev. B, 84, 094305 (2011).

[15] T. Brunet, S. Raffy, B. Mascaro, J. Leng, R. Wunenburger, O. Mondain-Monval, O. Poncelet, and C. Aristegui, Appl. Phys. Lett., 101, 011913 (2012).

[16] D. Dendukuri and P. S. Doyle, Adv. Mater., 21, 4071 (2009)

[17] A. S. Utada, E. Lorenceau, D. R. Link, P. D. Kaplan, H. A. Stone, and D. A. Weitz, Science, 308, 537 (2005)

[18] S. Afkhami, A. J. Tyler, Y. Renardy, M. Renardy, T. G. St. Pierre, R. C. Woodward, and J. S. Riffle, J. Fluid Mech., 663, 358 (2010)

[19] Further details will be published elsewhere.

[20] The longest wavelength involved in our acoustic experiments was lower than $1 \mathrm{~mm}$, i.e., at least 15 times lower 
than the transducer diameter.

[21] B. Mascaro, T. Brunet, O. Poncelet, C. Aristegui, S. Raffy, O. Mondain-Monval, and J. Leng, J. Acoust. Soc. Am., 133, 1996 (2013).

[22] A. Derode, V. Mamou, and A. Tourin, Phys. Rev. E, 74, 036606 (2006).

[23] A. Ishimaru, Wave propagation and scattering in random media (Academic Press, 1978).

[24] C. Flammer, Spheroidal Wave Functions (Dover, 2005).

[25] C. Yeh, J. Acoust. Soc. Am., 42, 518 (1967).
[26] W. M. Zhu, A. Q. Liu, X. M. Zhang, D. P. Tsai, T. Bourouina, J. H. Teng, X. H. Zhang, H. C. Guo, H. Tanoto, T. Mei, G. Q. Lo, and D. L. Kwong, Adv. Mater., 23, 1792 (2011).

[27] Z. Liu, X. Zhang, Y. Mao, Y. Y. Zhu, Z. Yang, C. T. Chan, and P. Sheng, Science, 289, 1734 (2000).

[28] T. Brunet, J. Leng, and O. Mondain-Monval, Science, 342, 323 (2013). 